Research Article

\title{
Prediction of Acoustic Energy Radiated by Bubble Produced by Raindrops
}

\author{
Qi Li, ${ }^{1,2,3}$ Shu Liu $\mathbb{D}^{1,2,3}$ and Dajing Shang $\mathbb{D}^{1,2,3}$ \\ ${ }^{1}$ Acoustic Science and Technology Laboratory, Harbin Engineering University, Harbin 150001, China \\ ${ }^{2}$ Key Laboratory of Marine Information Acquisition and Security (Harbin Engineering University), \\ Ministry of Industry and Information Technology, Harbin 150001, China \\ ${ }^{3}$ College of Underwater Acoustic Engineering, Harbin Engineering University, Harbin 150001, China \\ Correspondence should be addressed to Dajing Shang; shangdajing@hrbeu.edu.cn
}

Received 27 May 2020; Revised 11 November 2020; Accepted 4 December 2020; Published 14 December 2020

Academic Editor: Parviz Ghadimi

Copyright (C) 2020 Qi Li et al. This is an open access article distributed under the Creative Commons Attribution License, which permits unrestricted use, distribution, and reproduction in any medium, provided the original work is properly cited.

\begin{abstract}
Underwater noise produced by rainfall is an important part of underwater ambient noise. The bubbles produced by raindrops are the main noise source of underwater noise. Generally, the sound pressure signal of individual bubbles is easily contaminated by tank reverberation, hydrodynamic flow, and laboratory electrical noise. In order to solve this problem, this study proposes a method for calculating the acoustic energy of the bubble produced by a raindrop when the latter falls onto a plane water surface. For this purpose, a series of experiments was conducted in a $15 \mathrm{~m} \times 9 \mathrm{~m} \times 6 \mathrm{~m}$ reverberation tank filled with tap water. The bubble produced by a raindrop behaves as a simple exponentially damped sinusoidal oscillator. Based on the dipole radiation pattern, a formula was derived to predict the sound energy of these bubbles. The damping coefficient of the bubble formed by raindrops is found to differ appreciably from the empirical value of the bubble formed by other mechanisms. The resonance frequency of the bubbles is found to decrease with time. It is due to the rapid increase in the distance between the bubble and the interface. Then, the formula is optimized by using these two improved variables. The experimental results agree well with the theoretical derivation.
\end{abstract}

\section{Introduction}

Wind-generated and rain-generated ambient sound from the ocean surface represents the background baseline of ocean noise. Some studies have shown that when present, the sound produced by rainfall dominates the underwater sound field [1-3]. Two distinctive acoustic fields associated with rainfall have been described. The first, associated with light rain or drizzle, is characterized by a broad spectral peak near $15 \mathrm{kHz}$ [2]. The second, associated with heavy rainfall, is nearly white (from $4-20 \mathrm{kHz}$ ) and is highly correlated with the rainfall rate [3]. The study of rainfall noise is important not only for ocean physicists studying (i) rainfall and wind over the ocean [4] and (ii) the usage of sonars [5-7] but also for biologists studying how anthropogenically generated sound impacts marine mammals [8]. Another important application of rainfall (bubble) noise is in the field of biogeochemistry, specifically in estimating the rate of exchange of gas between air and water [9] or sediments and water [10].

The bubbles produced by raindrops are the main noise source of underwater noise due to rain. The dynamics of underwater bubbles have long been of considerable interest because of their importance in various physical and engineering problems, including cavitation on ship propellers [11-13], underwater explosions [14-16], and ultrasonic cleaning [17-19]. Although there has been some work on underwater bubble swarms $[20,21]$, there has been much less on the acoustic energy generated by bubbles produced by rainfall.

The earliest reference to bubbles as sound sources was made by Leonardo Da Vinci, who described the sound of running water already in the $1500 \mathrm{~s}$. Bragg [22] has attributed the murmuring of a brook and the "plunk" of droplets 
falling into water to entrained air bubbles. Minnaert [23] has since shown that the sound generated by gas bubbles in liquids is associated with simple volume pulsations of the bubble with no change of its shape. Minnaert then derived the resonance frequency of the bubble, which behaves as a simple exponentially damped sinusoidal oscillator with one degree of freedom. The number of cycles required for the amplitude of motion to reduce to $e^{-\pi}$ of its original value is known as the $Q$ factor of the bubble system. The total damping coefficient $\delta$ is now defined as the reciprocal $Q$ of the bubble system. Devin [24] has since shown that the damping may be explained by losses originating from three processes, namely, (i) thermal damping due to the thermal conduction between the gas in the bubble and the surrounding liquid, (ii) sound-radiation damping, and (iii) viscous damping due to viscous forces at the gas-liquid interface. The total damping is the sum of these three processes.

Several attempts have been made to show what happens when a raindrop strikes a water surface. Worthington [25] made the first photographic study, and Franz [26], Laville [27], and Buckingham [28] identified two main sources of underwater sound, namely, (i) the initial impact sound as a sharp pulse and (ii) the bubble sound as a decaying sinusoid. Franz also observed that the impact sound and bubble sound seem to behave as simple dipole sources near the free surface. Furthermore, laboratory experiments have been conducted to describe the sound generated by a single raindrop [29-32]. These experiments identified four mechanisms for raindrop-generated sound, namely, (i) the initial impact, (ii) a bubble trapped by a small raindrop at the bottom of the impact crater (type I), (iii) a bubble trapped underwater by a turbulent jet created by the splash canopy of a large raindrop (type II), and (iv) a bubble created during the secondary splashes of drop aerosols thrown up during the initial raindrop impact (type III). In the case of small raindrops associated with light rain and drizzle, a bubble-entrapment mechanism has been described, namely, the pinch-off of the tip of the splash crater by surface tension and gravity $[29,33,34]$. The bubbles created by this mechanism are designated as being of type I [31]. The process whereby a type-I bubble forms is shown in Figure 1(a). The raindrop hits the water surface at time $t=0$, and the bubble is produced at $t=21.8 \mathrm{~ms}$. In heavier rainfall, larger raindrops are present. The process whereby a type-II bubble forms is shown in Figure 1(b). Frame 1 shows the drop immediately before impact. In frames 2-5, a canopy is formed when a spray of droplets is ejected by the upward-moving water mass. At the apex of the canopy, water convergence generates upward- and downward-moving turbulent jets of water. In frame 10, a bubble is trapped underwater as the downward-moving jet penetrates the crater bottom. Bubbles formed by this mechanism are defined as being of type II [31-33].

Medwin and Beaky [35] found that the resonance frequencies of all those bubbles decrease during the short oscillation times of the latter, and they tried to explain this change. One possibility is that the bubble moves away from the surface rapidly, with subsequent displacement due to an increase in the dipole axis. Another possibility is that the bubble is initially ellipsoidal and that its frequency decreases as it becomes spherical. However, to date, no studies have addressed how the bubble could move away from the surface so quickly in less than $2 \mathrm{~ms}$ (the bubbles produced by raindrops usually last no more than $2 \mathrm{~ms}$ ).

There has been a lot of study on the physical properties of bubbles, but little research has been done on the acoustic energy radiated by a single bubble produced by a raindrop. The noise radiated by bubbles is a transient signal, which is easily contaminated by tank reverberation, hydrodynamic flow, and laboratory electrical noise in the water tank.

In order to eliminate the contamination, this study proposes a method for predicting the acoustic energy of the bubble produced by a raindrop. Using this method, the sound energy can be accurately calculated by measuring the direct sound pressure amplitude and initial resonance frequency of the bubble.

\section{Methods}

2.1. Theory. The bubble is assumed to be spherical (or sufficiently close to spherical) so that, away from the free surface of the water, the resonance frequency of the bubble is given by the following equation [36]:

$$
f_{0}=\frac{1}{2 \pi a} \sqrt{\frac{3 \gamma P_{0}}{\rho}},
$$

where $a$ is the bubble radius, $P_{0}$ is the ambient pressure surrounding the bubble, $\rho=1000 \mathrm{~kg} / \mathrm{m}^{3}$ is the density of the surrounding water, and $\gamma$ is the ratio of the specific heat of air $(\gamma=1.4)$.

A near-surface oscillating bubble forms a phase-reversed image in the smooth, reflecting water-air interface, and thereby behaves as a dipole. The far-field theoretical radiation pressure of the bubble acting as a dipole [35] is proportional to $\cos \theta$, where $\theta$ is the angle perpendicular to the water surface. This sound-radiation model of bubbles is shown in Figure 2, where $R$ is the distance from the point of impact to the hydrophone, $R_{2}$ is the distance from the image to the hydrophone, and $R_{1}$ is the distance from the bubble to the hydrophone. In general, the pool used in the experiment can be considered a reverberation pool. When the direct sound received by the measuring point is equal to the reflected sound, the distance between the measuring point and the sound source is defined as the reverberation radius $r_{n}$. When the distance between the measuring point and the sound source is less than $r_{n}$, the received signal is dominated by the direct sound. When the distance is greater than $r_{n}$, the received signal is dominated by the reflected sound.

If $R$ is much less than the reverberation radius $r_{n}$, the reflected sound can be ignored. Then, the energy radiated by a surface dipole [32] is given by the following equation:

$$
E=\frac{2 \pi R^{2}}{3 \rho c} \int p_{\mathrm{ax}}^{2}(t) \mathrm{d} t,
$$

where $R$ is the distance from the point of impact to the hydrophone, $c=1500 \mathrm{~m} / \mathrm{s}$ is the speed of sound in water, and 

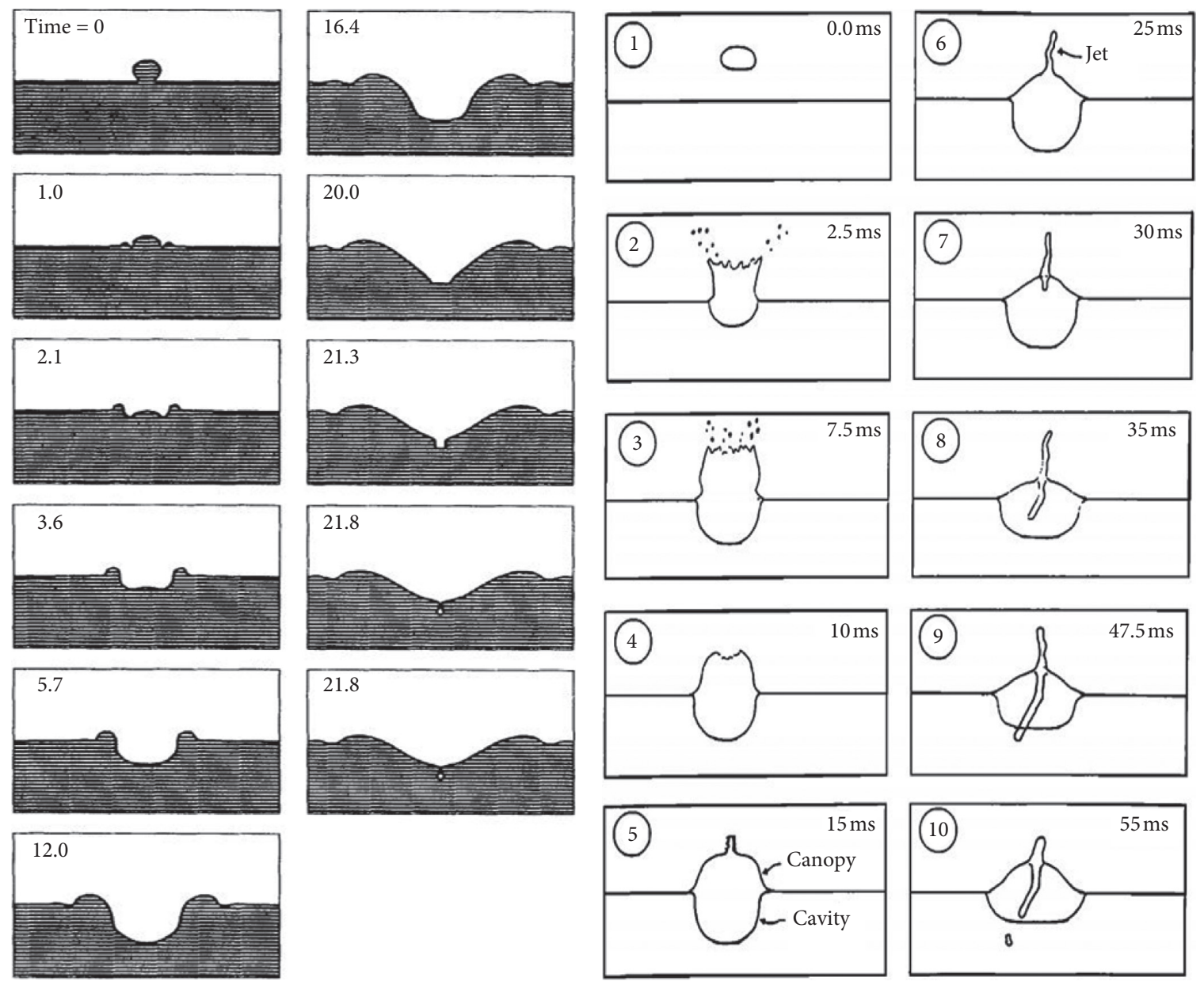

(b)

Figure 1: Physical sequences of raindrop splashes: (a) small raindrop splash (redrawn from Figure 9 in [34]); (b) large raindrop splash (redrawn from Figure 3 in [32]).

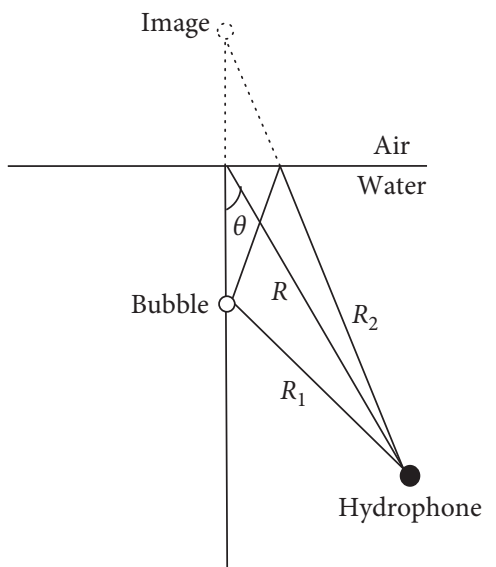

FIgURE 2: Sound-radiation model of a bubble near the surface.

$p_{\text {ax }}(t)$ is the axial acoustic pressure at range $R$. The acoustic energy can be calculated using equation (2) once $p_{\mathrm{ax}}(t)$ has been measured. The acoustic pressure signal is a transient signal, which is easily contaminated by tank reverberation, hydrodynamic flow, and laboratory electrical noise in the water tank. To eliminate the contamination, the acoustic energy of a bubble can be predicted as follows.

The acoustic pressure signal is described by a damped sinusoid, namely,

$$
p_{\mathrm{ax}}(t)=p_{0} \sin \left(2 \pi f_{0} t\right) e^{-2 \pi f_{0} \delta t}
$$

where $p_{0}$ is the peak pressure at $R_{0}=1 \mathrm{~m}$ (the distance from the point of impact to the hydrophone) on axis (based on the dipole radiation pattern) and $\delta$ is the damping coefficient for an air bubble in water [36] and is approximated by

$$
\delta \cong k \cdot\left(\frac{f_{0}}{1000}\right)^{1 / 3} \text {. }
$$

This is an empirical formula for the damping coefficient of bubbles produced by the bubble generator. Also, $k=0.025$ when the resonance frequency of the bubble is between 1 and $100 \mathrm{kHz}$.

Substituting equation (3) into equation (2) gives 


$$
\begin{aligned}
E & =\frac{2 \pi R_{0}^{2} p_{0}^{2}}{3 \rho c} \int \frac{1-\cos \left(4 \pi f_{0} t\right)}{2} e^{-4 \pi f_{0} \delta t} \mathrm{~d} t \\
& =\frac{2 \pi R_{0}^{2} p_{0}^{2}}{3 \rho c} \int \frac{1-\Re\left(e^{-4 j \pi f_{0} t}\right)}{2} e^{-4 \pi f_{0} \delta t} \\
\mathrm{~d} t & =\frac{R_{0}^{2} p_{0}^{2}}{12 \rho c f_{0} \delta\left(\delta^{2}+1\right)},
\end{aligned}
$$

which is the equation used to predict the energy radiated by a bubble produced by a raindrop. Here, because the damping coefficient is much less than unity according to equation (4), we have that $\delta^{2}+1 \approx 1$, and then, the formula can be simplified as follows:

$$
E=\frac{R_{0}^{2} p_{0}^{2}}{12 \rho c f_{0} \delta}
$$

2.2. Experiment. To verify the accuracy of equation (6) for predicting the acoustic energy radiated by a bubble produced by a raindrop, we conducted a series of experiments in a $15 \mathrm{~m} \times 9 \mathrm{~m} \times 6 \mathrm{~m}$ reverberation pool filled with tap water up to its maximum height $(6 \mathrm{~m})$. The temperature of water is 20 degree Celsius, and the temperature of air is 18.5 degree Celsius. Figure 3 shows the system used to measure the sound signal generated by raindrops. The four acoustically distinct ranges of the drop diameter $D$ are defined as (i) minuscule drops $(D \leq 0.8 \mathrm{~mm})$, (ii) small drops $(0.8 \mathrm{~mm}<D \leq 1.1 \mathrm{~mm})$, (iii) midsize drops $(1.1 \mathrm{~mm}<D \leq 2.2 \mathrm{~mm})$, and (iv) large drops $(D>2.2 \mathrm{~mm})$. We used a standard intravenous drip bottle with needles of different caliber to generate drops of tap water of five different diameters, namely, $0.7,0.9,1.5,2.5$, and $3.9 \mathrm{~mm}$. The height of the drop bottle is five meters. At this height, each drop struck the water surface at the terminal velocity of the raindrops in natural rainfall. Various testing conditions are listed in Table 1.

We suspended a hydrophone (type 8103; Brüel and Kjær, Denmark) $20 \mathrm{~cm}$ below the impact point. This distance is much less than the pool's reverberation radius $r_{n}=1.7 \mathrm{~m}$ measured by Dajing et al. [37]. At this distance, the effect of reflected sound can be avoided. The hydrophone signal was sent to a computer using a pulse collector (type 3560E; Brüel and Kjær). Meanwhile, high-speed video photography was used to observe the underwater movement of the bubble produced by the drop. The camera (HERO4; GoPro, USA) operated at 240 frames per second (fps) and was placed underwater near the impact point along with a ruler to measure the distance of the bubble from the surface. For each bubble, the result of this procedure was a single film comprising a sequence of photographs of the bubble in the water.

\section{Results and Discussion}

3.1. Acoustic Energy of the Bubbles. The $0.7 \mathrm{~mm}$ drop produced only weak impact-pulse noise. The $0.9 \mathrm{~mm}$ drop produced weak impact-pulse noise and a type-I damped bubble. The $1.5 \mathrm{~mm}$ drop produced only impact-pulse noise.
The $2.5 \mathrm{~mm}$ and $3.9 \mathrm{~mm}$ drops both produced impact-pulse noise and type-II and type-III damped bubbles.

Using the physical mechanisms for the three types of bubble produced by raindrops, we clarify here why the $1.5 \mathrm{~mm}$ drop produces only impact noise and no bubble, whereas the $0.9 \mathrm{~mm}$ and $2.5 \mathrm{~mm}$ drops both produce bubbles. Because the resultant force of gravity and surface tension is not strong enough to pinch-off the tip of the splash crater, there is no type-I bubble when a midsize raindrop strikes the water surface. Because a midsize raindrop does not have enough kinetic energy, no canopy is formed when the upward-moving water mass ejects a spray of droplets, and no bubble is trapped underwater. There is no type-II bubble when a midsize raindrop strikes the water surface, nor is there a type-III bubble because there are no secondary splashes of drop aerosols thrown up during the initial raindrop impact.

The sound pressure signal collected $20 \mathrm{~cm}$ below the impact point is converted to sound pressure at $1 \mathrm{~m}$ on axis according to the dipole radiation pattern. Figure 4(a) shows the acoustic signal produced by the raindrop of $3.9 \mathrm{~mm}$ in diameter. As can be seen in Figure 4(a), the signal generated by the initial pulse is measured at time $t=7.3 \mathrm{~ms}$, and the signal generated by the bubble is measured at time $t=52.4 \mathrm{~ms}$. The bubble is detected around $45.1 \mathrm{~ms}$ after impact. The sinusoidally damped bubble signal in Figure 4(a) is shown in Figure 4(b) with an appropriate amplitude amplification. Here, the echo is clearly distinguished from direct sound.

The amplitudes of the impact-pulse noise and bubble noise generated by raindrops are given in Table 2 , where $D$ is the raindrop diameter, $p_{P}$ is the amplitude of the impactpulse noise, and $p_{\mathrm{I}}, p_{\mathrm{II}}$, and $p_{\mathrm{III}}$ are the amplitudes of type I, II, and III bubble noise, respectively. The amplitude of the impact-pulse noise generated by raindrops of the same diameter is clearly uniform and increases with the raindrop diameter. The average amplitude of the impact-pulse noise generated by raindrops of $2.5 \mathrm{~mm}$ and $3.9 \mathrm{~mm}$ in diameter is, respectively, $0.65 \mathrm{~Pa}$ and $2.02 \mathrm{~Pa}$. The amplitude distribution of the type-I bubble noise is concentrated and uniform. The average amplitude of the type-I bubble noise is $0.36 \mathrm{~Pa}$, whereas the amplitude distributions of the type-II and typeIII bubble noise are dispersive and random. As the raindrop diameter increases, more bubbles with high sound pressure are produced.

The actual acoustic energy of the bubble was calculated using equation (2), while the theoretical acoustic energy of the bubble was calculated using equation (6). $p_{0}$ is the peak pressure at the distance $R_{0}=1 \mathrm{~m}$ from the point of impact to the hydrophone.

Figure 5 compares the actual acoustic energy and the theoretical values for the three types of bubble (for the convenience of comparison, the actual sound energy data are sorted from smallest to largest). The theoretical acoustic energy of type-I bubble is less than the experimental value by about $60 \%$ overall. The root mean square (RMS) of the error is $1.50 \times 10^{-11} \mathrm{~J}$. The theoretical acoustic energy of type-II and type-III bubbles is less than the experimental value by about $50 \%$ overall. The root mean square of the errors is 


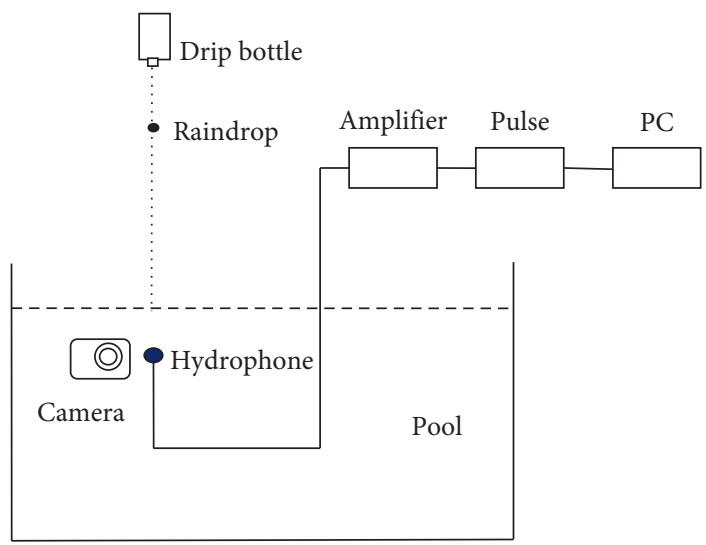

Figure 3: A system used to measure sound signal generated by raindrops.

TABLE 1: Testing conditions for bubbles produced by raindrops.

\begin{tabular}{lcccc}
\hline No. & $D(\mathrm{~mm})$ & Drop type & Number of tests & Bubble type and probability \\
\hline 1 & 0.7 & Minuscule & 50 & No bubble \\
2 & 0.9 & Small & 62 & Type I (100\%) \\
3 & 1.5 & Midsize & 50 & No bubble \\
4 & 2.5 & Large & 92 & Type II (28.3\%), type III (34.8\%) \\
5 & 3.9 & Large & 94 & Type II (57.4\%), type III (21.2\%) \\
\hline
\end{tabular}

No. is the serial number. $D$ is the raindrop diameter.

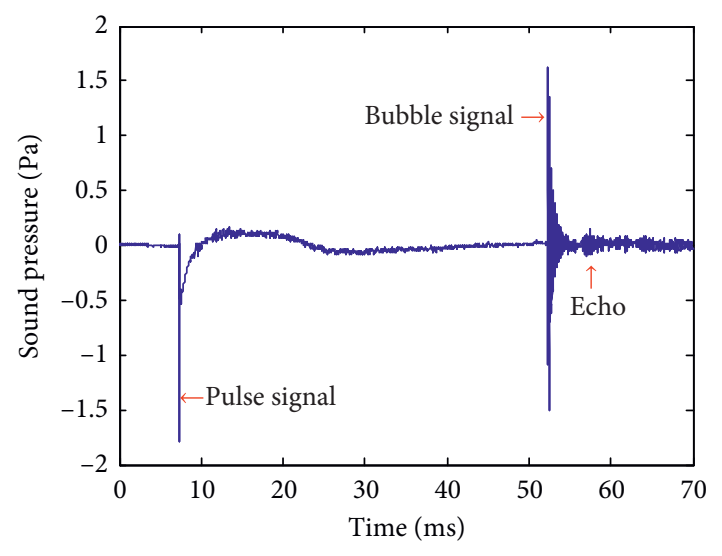

(a)

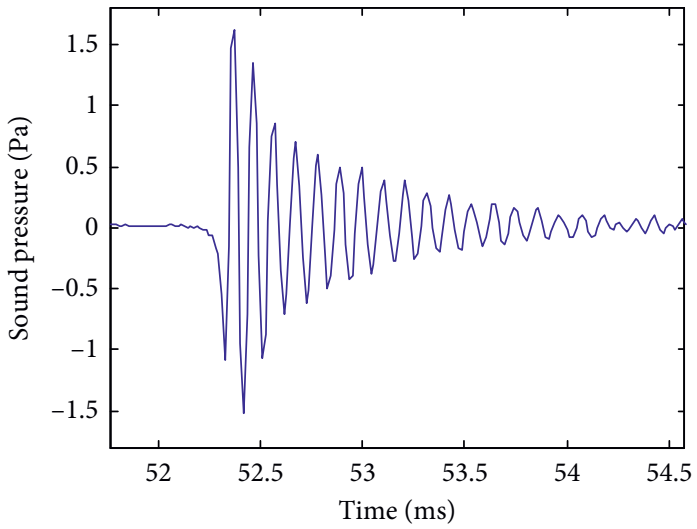

(b)

Figure 4: Acoustic signal produced by a raindrop of $3.9 \mathrm{~mm}$ in diameter: (a) entire signal; (b) sinusoidally damped bubble signal.

TABLE 2: Ranges of amplitude of sound pressure.

\begin{tabular}{lcccc}
\hline$D(\mathrm{~mm})$ & $p_{P}(\mathrm{~Pa})$ & $p_{\text {I }}(\mathrm{Pa})$ & $p_{\text {II }}(\mathrm{Pa})$ & - \\
\hline 0.9 & - & $0.25-0.54$ & - & $p_{\text {III }}(\mathrm{Pa})$ \\
2.5 & $0.51-0.74$ & - & $0.08-1.4$ & - \\
3.9 & $1.82-2.44$ & - & $0.06-2.0$ & $0.06-1.22$ \\
\hline
\end{tabular}

$2.17 \times 10^{-10} \mathrm{~J}$ and $3.55 \times 10^{-10} \mathrm{~J}$, respectively. According to equation (6), the deviation of acoustic energy may be caused by the deviation of damping coefficient $\delta$ or resonance frequency $f_{0}$.
3.2. Influence of Damping Coefficient. The empirical damping coefficient in equation (4) is used to calculate the theoretical acoustic energy of bubble produced by the bubble generator. The actual damping coefficient $\delta$ of each bubble 


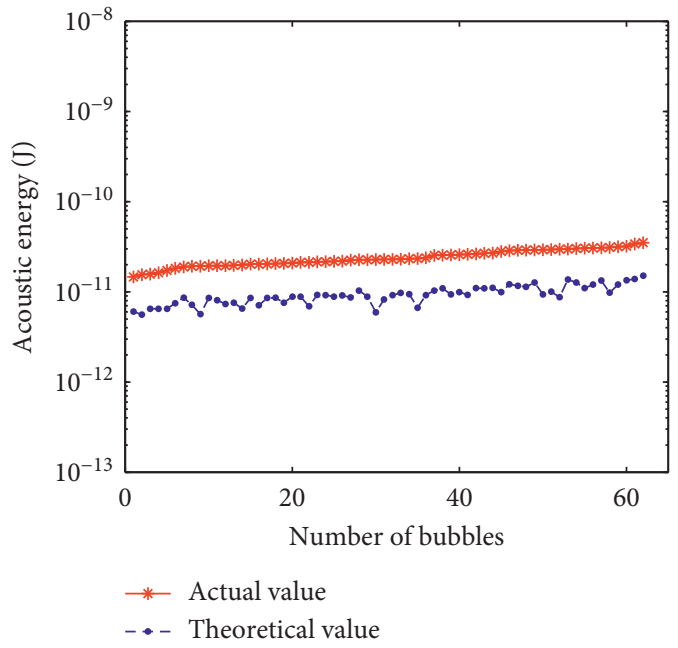

(a)

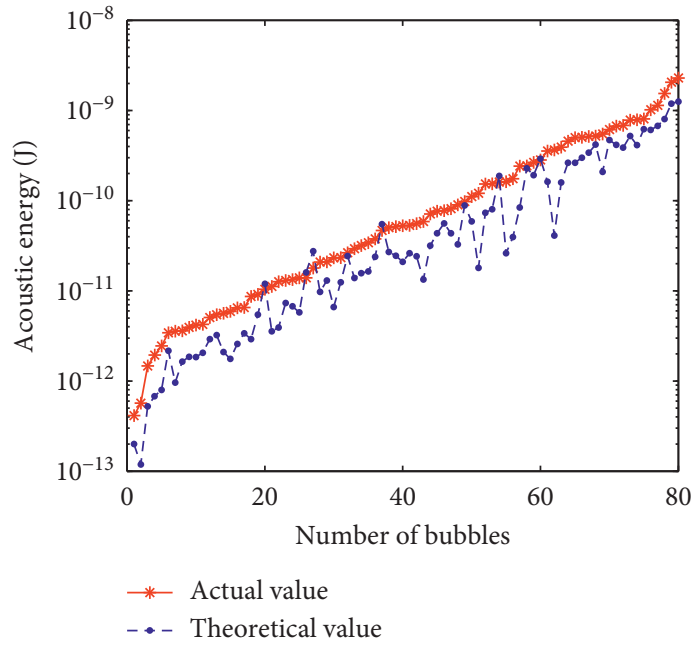

(b)

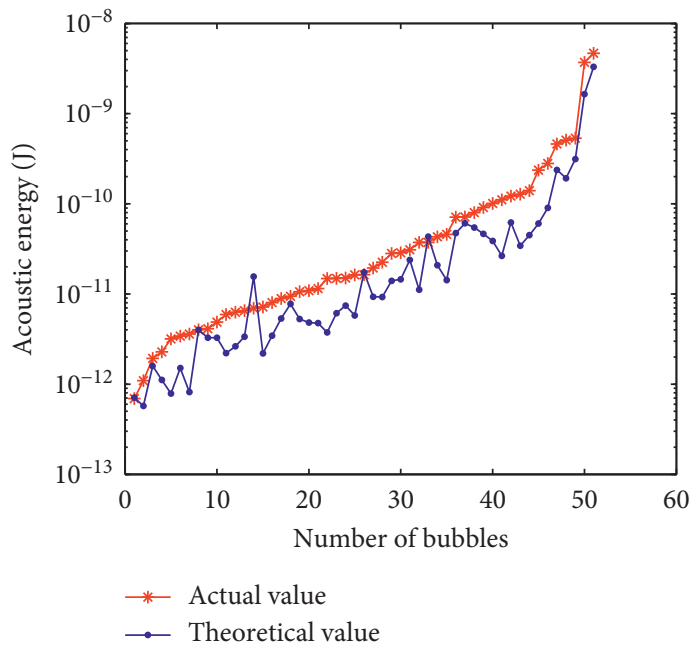

(c)

Figure 5: Actual acoustic energy of bubble calculated using equation (2) (red) and theoretical acoustic energy of bubble calculated using equation (6) (blue): (a) type-I bubble; (b) type-II bubble; (c) type-III bubble.

produced by each size of drop in the present experiments is fitted using equation (3). The actual damping coefficient and empirical damping coefficient are shown in Figure 6. The actual damping coefficients are different from the empirical values. The reason is that the bubbles produced by rainfall are different in mechanism from those produced by bubble generators. Bubbles generated by bubble generators are just beginning to be static in water. Then, the bubbles rise slowly under the influence of buoyancy. However, as soon as the bubbles are produced by raindrops, they will move down rapidly under the influence of inertia. The heat generated by the relative moving between the bubbles and water reduces the outward thermal radiation of the bubbles. The thermal damping of the bubbles produced by raindrops is less than that of other origin. Therefore, the actual damping coefficients are found to be less than the empirical values. Gillot et al. [38] have pointed out that the values of the damping time obtained by theoretical predictions are far from the values measured in water. This deviation of damping coefficient $\delta$ results in the deviation of acoustic energy.

Therefore, a new empirical formula for the damping coefficient of an air bubble produced by a raindrop is given by

$$
\delta=k_{1} \cdot\left(\frac{f_{0}}{1000}\right)^{1 / 3}
$$

where $k_{1}=0.012$. This is the empirical formula for the damping coefficient of bubbles produced by raindrops. The empirical damping coefficient in equation (4) is twice the actual damping coefficient. The new curve fitted is shown in Figure 6.

The theoretical acoustic energy of the bubble was recalculated using the modified damping coefficient. Figure 7 compares the actual acoustic energy and the corrected theoretical values for the three types of bubble. In each case, the 


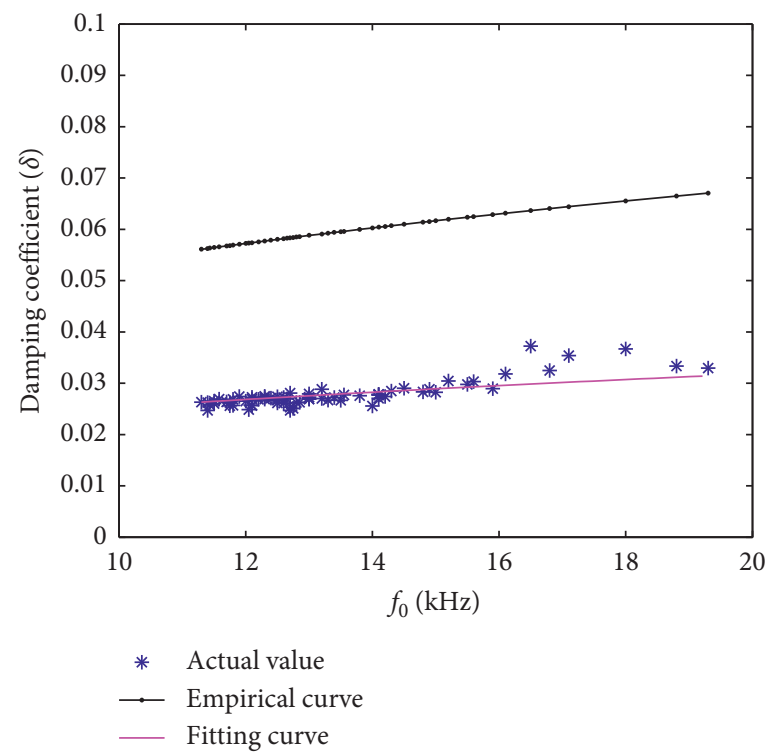

Figure 6: Comparison of the actual damping coefficient of bubbles produced by raindrops with the empirical value.

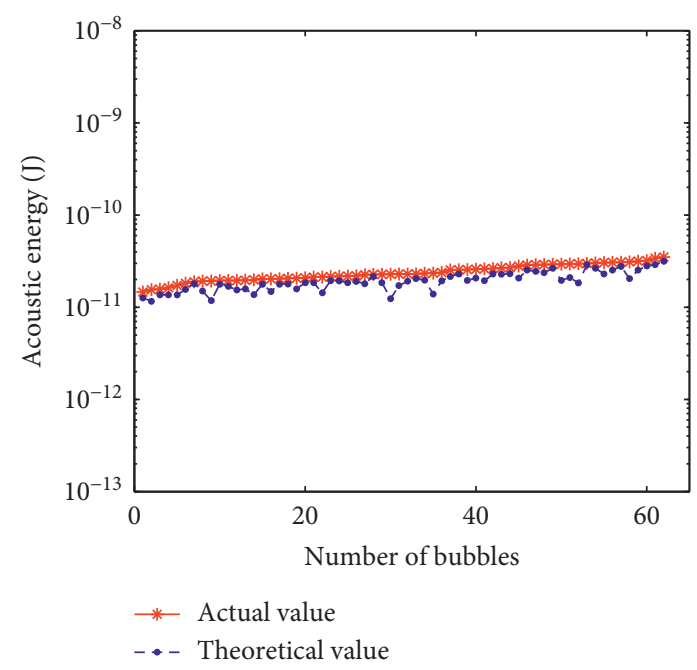

(a)

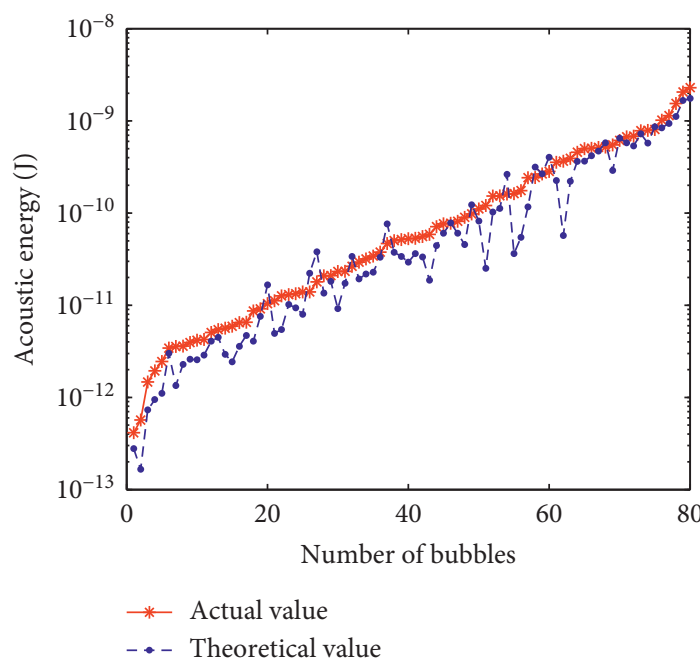

(b)

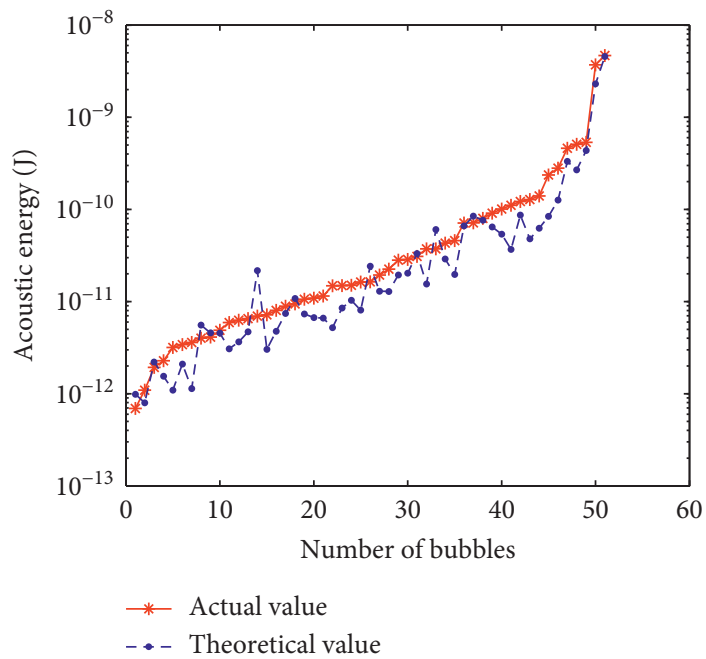

(c)

Figure 7: Actual acoustic energy of bubble calculated using equation (2) (red) and correctional theoretical acoustic energy of bubble calculated using equation (6) (blue): (a) type-I bubble; (b) type-II bubble; (c) type-III bubble. 
theoretical acoustic energy is very close to the experimental value, but the theoretical value is still less than the experimental value by about $10 \%$ overall. The root mean square of the errors is $4.87 \times 10^{-12} \mathrm{~J}, 1.19 \times 10^{-10} \mathrm{~J}$, and $2.05 \times 10^{-10} \mathrm{~J}$, respectively.

3.3. Influence of Resonance Frequency. The corrected theoretical values for the three types of bubble are still less than the experimental value by about $10 \%$ overall in Section 3.2. According to equation (6), in addition to damping coefficient, the bubble pulsation frequency may also affect the acoustic energy radiated by the bubble. In order to study the influence of bubble resonance frequency on bubble acoustic energy, the actual bubble acoustic pressure curve is analyzed. The sound pressure signal collected by the hydrophone was Fourier transformed to obtain the initial resonance frequency $f_{0}$ of the bubble, and then, the actual damping coefficient $\delta$ of the bubble was calculated according to equation (3). According to the initial resonance frequency and damping coefficient, the sound pressure curve of the bubble is fitted.

Figure 8 compares the actual sound pressure curve (red) and fitting sound pressure curve (blue). The fitting curve is an exponentially damped sinusoidal curve. As can be seen from the figure, the actual curve completely coincides with the fitting curve in the first few cycles. After a few cycles, the two curves begin to separate. The period of the actual curve gets longer, which means the resonance frequency of the bubble is decreasing. As a result, the theoretical acoustic energy calculated using equation (6) is lower than the actual value.

Medwin and Beaky [35] found that the resonance frequencies of all those bubbles decrease during the short oscillation times of the latter, and they tried to explain this change. One possibility is that the bubble is initially ellipsoidal and that its frequency decreases as it becomes spherical. Strasberg [39] proved that the frequency is only slightly dependent on $e$ ( $e$ is the ratio of major to minor axis of the spheroid). For example, the frequency of an oblate spheroid with $e=2$ differs by only 2 percent from that of a sphere with the same volume. Because actually $e$ is less than 2 , the deviation is less than 2 percent. Another possibility is that the bubble moves away from the surface rapidly, with subsequent displacement due to an increase in the dipole axis. However, to date, no studies have addressed how the bubble could move away from the surface so quickly in less than $2 \mathrm{~ms}$ (the bubbles produced by raindrops usually last no more than $2 \mathrm{~ms}$ ). In view of this, this paper puts forward an explanation as follows.

3.4. Explanation for Decrease in Resonance Frequency. Figure 9 shows the process of bubble generation and movement underwater as recorded by the underwater camera running at $240 \mathrm{fps}$, showing a drop of $3.9 \mathrm{~mm}$ in diameter impacting at terminal velocity. The frames are in order, and the final three are sequential. The total time is around $71 \mathrm{~ms}$. The initial impact sound occurs in frame 1 (Figure 9(a)), a turbulent jet is created by the splash in frame 16 (Figure 9(b)), and a bubble is trapped underwater by the turbulent jet in frames 17 (Figure 9(c)) and 18 (Figure 9(d)). The bubble sound lasting less than $4.2 \mathrm{~ms}$ is detected between frames 17 and 18 around $67 \mathrm{~ms}$ after impact; it begins at the moment when the bubble detaches.

The bubble is connected to the atmosphere when it is generated at the bottom of the turbulent jet in frame 17 (also in frame 9 of Figure 1(a) and in frame 9 of Figure 1(b)). Although the bottom of the jet is $9 \mathrm{~mm}$ from the water surface, the depth of the bubble is considered to be zero when the bubble is connected to the atmosphere. The depth is equal to the diameter of the bubble at the moment when the bubble separates from the jet. After this separation, the bubble keeps going down, while water fills the crater. The depth of the bubble increases sharply in a time that is less than the $4.2 \mathrm{~ms}$ time interval between the two adjacent frames 17 and 18. Although the duration of the bubble sound is less than $4.2 \mathrm{~ms}$, the depth of the bubble increases by around $9 \mathrm{~mm}$ during the bubble pulsation period.

For a spherical bubble at close separation $z$ from the free surface, Strasberg [39] indicated that the resonance frequency will be higher, namely,

$$
f=f_{\infty} F
$$

where $f_{\infty}$ is the resonance frequency of the bubble in free field and $F$ is a depth-dependent factor as follows:

$$
F=\left[1-\left(\frac{a}{2 z}\right)-\left(\frac{a}{2 z}\right)^{4}\right]^{-(1 / 2)},
$$

where $z$ is the depth of the bubble. Gillot et al. [38] have calculated the corrected frequency using the correction coefficients due to the deformation of the bubble and to the proximity to the interface, respectively.

Figure 10 shows the relationship between the factor $F$ and the bubble depth for the initial pulsation frequencies of $5 \mathrm{kHz}, 15 \mathrm{kHz}$, and $25 \mathrm{kHz}$. In all three cases, $F$ decreases by around $10 \%$ when the bubble depth increases to ten times the radius of the bubble from the initial depth, which means that the bubble's pulsation frequency also decreases by $10 \%$. The minimum depth of the bubble is its radius. Meanwhile, the bubble frequency decreases more rapidly as the bubble's initial pulsation frequency is increased. In Figure 7, the theoretical acoustic energy is less than the experimental value by $10 \%$ overall, which is consistent with the trend associated with the decrease in the bubble's pulsation frequency. This consistency indicates that the decrease in the bubble's pulsation frequency results in the deviation of the acoustic energy. 


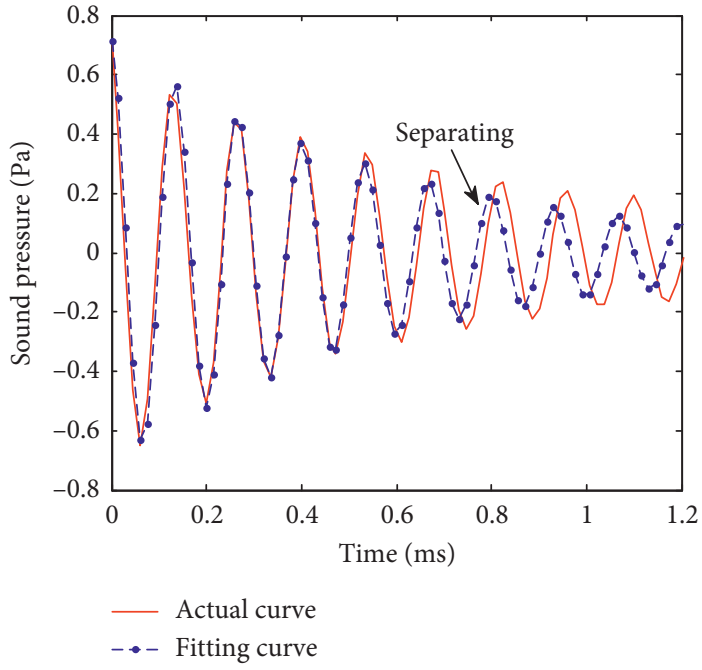

(a)

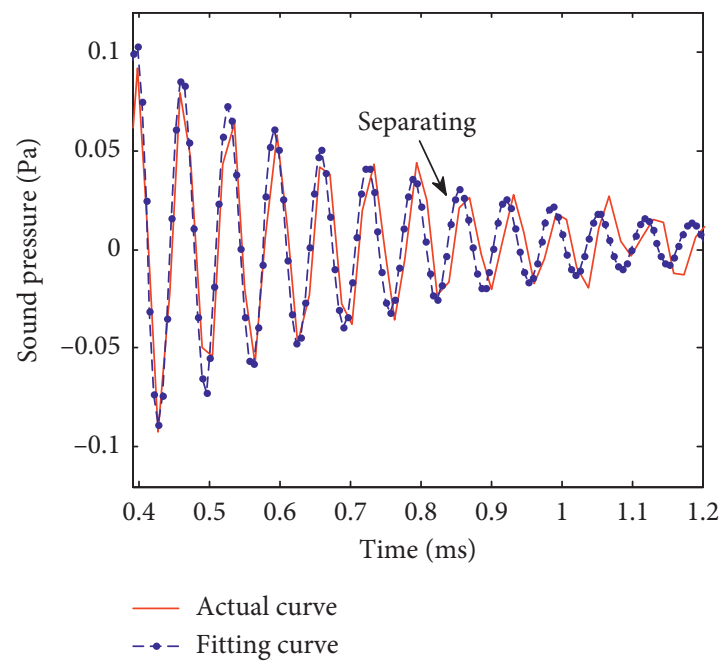

(b)

Figure 8: Actual sound pressure curve (red) and fitting sound pressure curve (blue): (a) $f_{0}=7.5 \mathrm{kHz}$; (b) $f_{0}=15 \mathrm{kHz}$. The fitting curve is an exponentially damped sinusoidal curve.

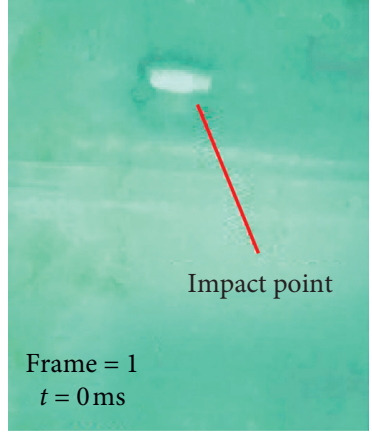

(a)

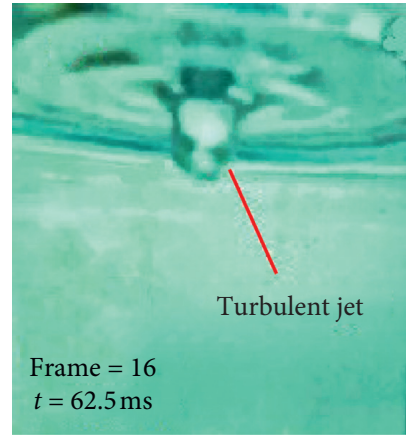

(b)

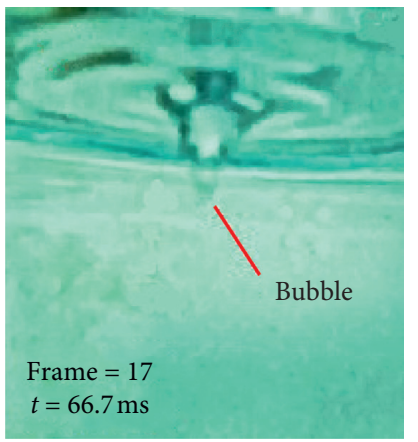

(c)

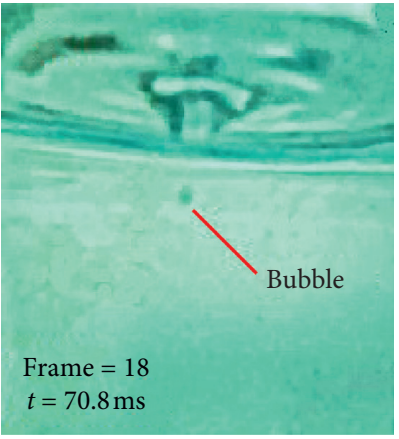

(d)

Figure 9: Frames from the underwater camera running at $240 \mathrm{fps}$ showing a drop of $3.9 \mathrm{~mm}$ in diameter impacting at terminal velocity. The time interval between each frame is approximately $4.2 \mathrm{~ms}$.

3.5. The Final Prediction Formula. Considering the correction of damping coefficient and decrease in the bubble's resonance frequency, the prediction formula of sound energy is as follows:

$$
E=\frac{R_{0}^{2} p_{0}^{2}}{12 \rho c f \delta}
$$

where $p_{0}$ is the peak pressure at $R_{0}=1 \mathrm{~m}$ on axis and $\delta$ is the damping coefficient in equation (7). Also, $f=\left(f_{0}+f_{1}\right) / 2$, $f_{0}$ is the initial resonance frequency of the bubble, and $f_{1}$ is the resonance frequency of the last cycle of the sinusoidally damped bubble signal. The resonant frequency is modified by adding a factor of 0.9 , which makes the theoretical sound energy consistent with the actual sound energy. In practice, $f$ approximates to $0.9 f_{0}$. Figure 11 compares the actual acoustic energy and the theoretical values calculated using final prediction formula equation (10) for the three types of bubble. In each case, the theoretical acoustic energy is very close to the experimental value. The RMS of the errors of bubble sound energy of types I, II, and III reduced from $1.50 \times 10^{-11} \mathrm{~J}$, $2.17 \times 10^{-10} \mathrm{~J}$, and $3.55 \times 10^{-10} \mathrm{~J}$ to $3.25 \times 10^{-12} \mathrm{~J}$, $9.11 \times 10^{-11} \mathrm{~J}$, and $1.78 \times 10^{-10} \mathrm{~J}$, respectively. 


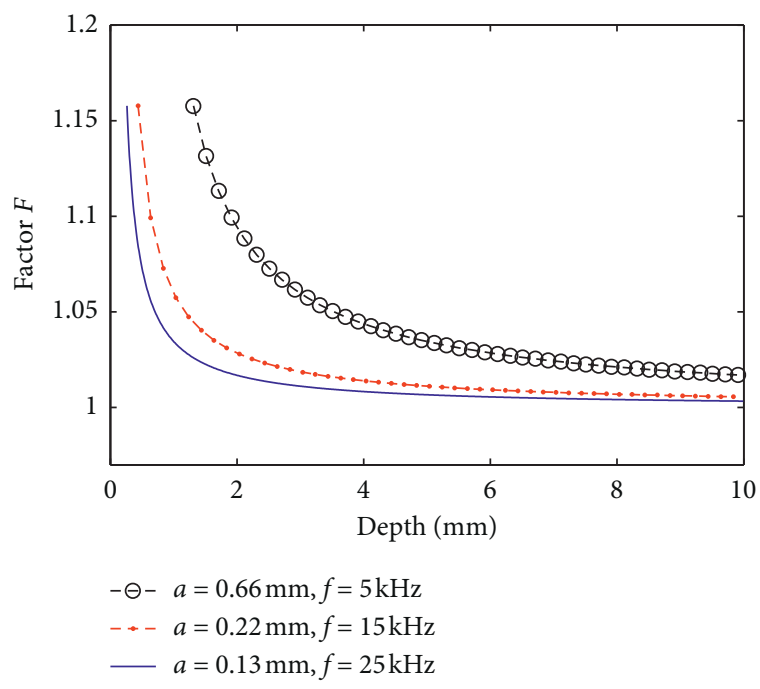

FIgURE 10: Variation in factor $F$ in equation (9) with bubble depth for different initial pulsation frequencies. The initial depth is the diameter of the bubble.

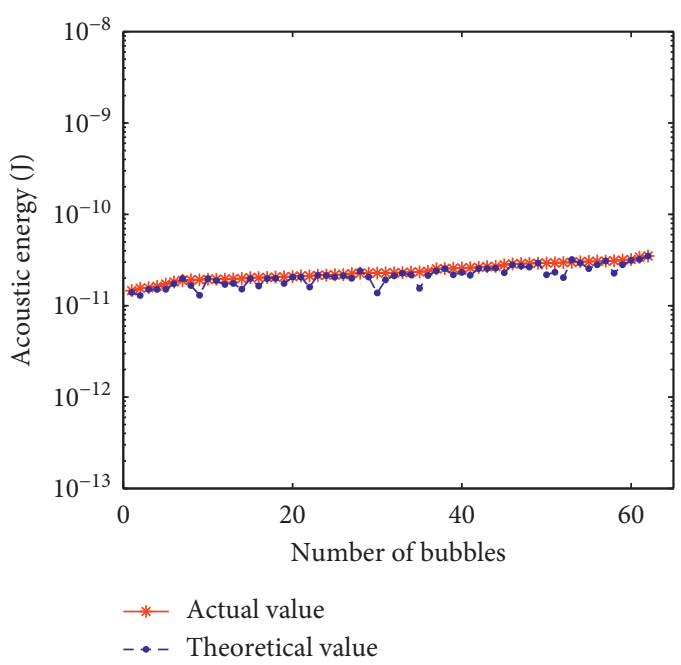

(a)

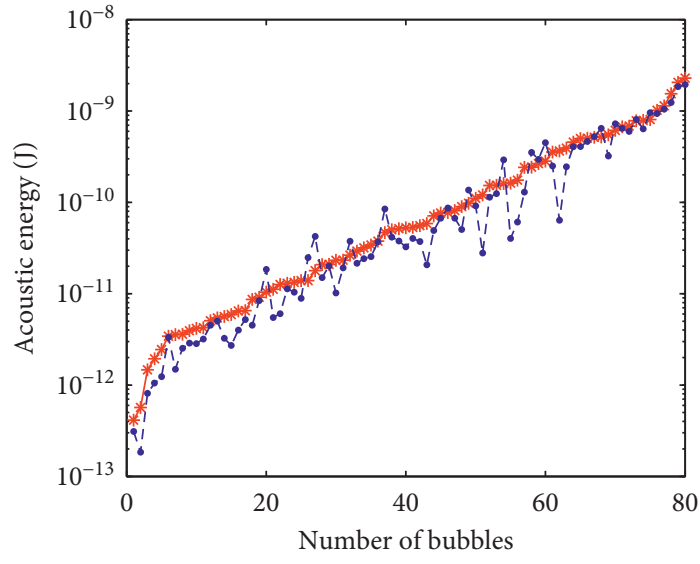

$\rightarrow$ Actual value

- - Theoretical value

(b)

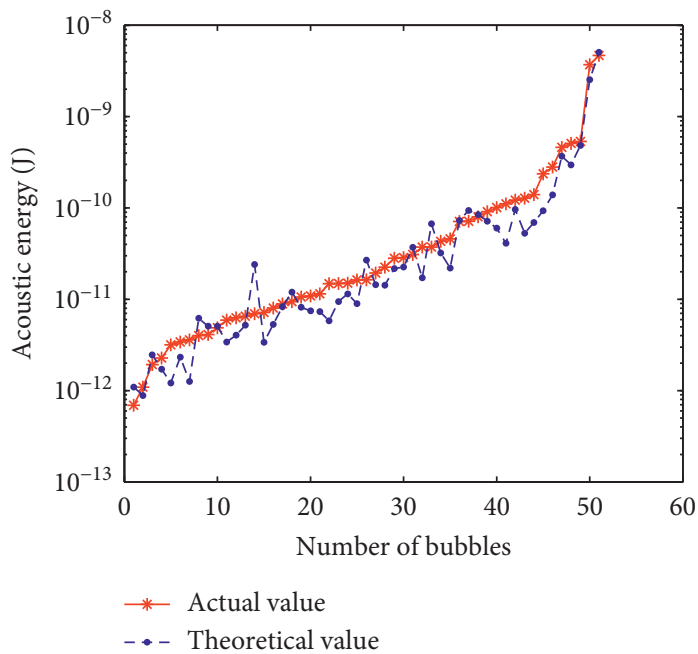

(c)

Figure 11: Actual acoustic energy of bubble calculated using equation (2) (red) and theoretical acoustic energy of bubble calculated using equation (10) (blue): (a) type-I bubble; (b) type-II bubble; (c) type-III bubble. 


\section{Conclusion}

In order to eliminate the contamination from the tank reverberation, hydrodynamic flow, and laboratory electrical noise in the water tank, a formula has been proposed for predicting the acoustic energy of the bubble produced by a raindrop when the latter falls onto a plane water surface. In order to verify and improve this formula, a series of experiments was conducted. By filming the impact with a highspeed underwater camera and recording the sound with a hydrophone $20 \mathrm{~cm}$ below the impact point, the acoustic energy radiated by the bubble produced by raindrops of different sizes has been studied. The main findings of this study are as follows:

(1) The damping coefficient of the bubble produced by a raindrop is found to differ appreciably from the empirical value of the bubble produced by bubble generators. The empirical damping coefficient of bubbles is twice the actual value. The reason is that the bubbles produced by rainfall are different in mechanism from those produced by bubble generators. This deviation of damping coefficient $\delta$ results in a $50 \%$ deviation between the theoretical and actual values of acoustic energy in the formula.

(2) After the correction of damping coefficient of the bubble, there is still $10 \%$ deviation between the theoretical and actual values of acoustic energy in the formula. This deviation is caused by the decrease in resonance frequency $f_{0}$ of the bubble. The bubble moves away from the water surface, thereby decreasing its resonance frequency rapidly.

(3) After the correction of damping coefficient and resonance frequency of the bubble, the results predicted by the formula proposed in this paper for predicting the acoustic energy of the bubble produced by a raindrop agree well with the actual acoustic energy. The RMS of the errors of bubble sound energy of types I, II, and III reduced from $1.50 \times 10^{-11} \mathrm{~J}, 2.17 \times 10^{-10} \mathrm{~J}$, and $3.55 \times 10^{-10} \mathrm{~J}$ to $3.25 \times 10^{-12} \mathrm{~J}, 9.11 \times 10^{-11} \mathrm{~J}$, and $1.78 \times 10^{-10} \mathrm{~J}$, respectively. This formula can be used to accurately predict the sound energy of bubbles produced by rainfall, even if the sound pressure signal of individual bubbles is easily contaminated by tank reverberation, hydrodynamic flow, and laboratory electrical noise.

\section{Data Availability}

The data used to support the findings of this study are available from the corresponding author upon request.

\section{Conflicts of Interest}

The authors declare that there are no conflicts of interest regarding the publication of this paper.

\section{Acknowledgments}

This research was funded by the Acoustic Science and Technology Laboratory of Harbin Engineering University (Grant no. SSJSWDZC2018010) and by the National Natural Science Foundation of China (Grant no. 11874131).

\section{References}

[1] P. T. Shaw, D. R. Watts, and H. T. Rossby, "On the estimation of oceanic wind speed and stress from ambient noise measurements," Deep Sea Research, vol. 25, no. 12, pp. 1225-1233, 1978.

[2] J. A. Nystuen, "Rainfall measurements using underwater ambient noise," The Journal of the Acoustical Society of America, vol. 79, no. 4, pp. 972-982, 1986.

[3] J. A. Nystuen, C. C. McGlothin, and M. S. Cook, "The underwater sound generated by heavy rainfall," The Journal of the Acoustical Society of America, vol. 93, no. 6, pp. 3169-3177, 1993.

[4] J. A. Nystuen, S. E. Moore, and P. J. Stabeno, "A sound budget for the southeastern Bering Sea: measuring wind, rainfall, shipping, and other sources of underwater sound," The Journal of the Acoustical Society of America, vol. 128, no. 1, pp. 58-65, 2010.

[5] A. Juan, "Ambient noise inverted sonar performance sensitivity due to ocean variability," The Journal of the Acoustical Society of America, vol. 121, no. 5, p. 3055, 2007.

[6] A. Rami, S. Heba, and A. E. Mohamad, "A novel fractional Fourier transform-based ASK-OFDM system for underwater acoustic communications," Applied Sciences, vol. 7, no. 12, p. 1286, 2017.

[7] Q. Li, J. Xing, D. Shang, and Y. Wang, "A flow velocity measurement method based on a PVDF piezoelectric sensor," Sensors, vol. 19, no. 7, p. 1657, 2019.

[8] J. L. Miksis, P. L. Donaghay, J. H. Miller et al., "Noise level correlates with manatee use of foraging habitats," The Journal of the Acoustical Society of America, vol. 121, no. 5, pp. 3011-3020, 2007.

[9] M. Klaus, E. Geibrink, E. Hotchkiss et al., "Listening to airwater gas exchange in running waters," Limnology \& Oceanography, Methods, vol. 17, no. 7, pp. 395-414, 2019.

[10] T. G. Leighton and P. R. White, "Quantification of undersea gas leaks from carbon capture and storage facilities, from pipelines and from methane seeps, by their acoustic emissions," Proceedings of the Royal Society A: Mathematical, Physical and Engineering Sciences, vol. 468, no. 2138, pp. $485-510,2012$.

[11] J. R. Blake, Y. Tomita, and R. P. Tong, "The art, craft and science of modelling jet impact in a collapsing cavitation bubble," Applied Scientific Research, vol. 58, no. 1-4, pp. 77-90, 1997.

[12] B. Ji, X. Luo, R. E. A. Arndt, and Y. Wu, "Numerical simulation of three dimensional cavitation shedding dynamics with special emphasis on cavitation-vortex interaction," Ocean Engineering, vol. 87, pp. 64-77, 2014.

[13] D. J. Shang, Q. Li, D. J. Shang et al., "Experimental investigation on flow-induced noise of the underwater hydrofoil structure," Acta Acustica, vol. 37, pp. 416-423, 2012, in Chinese.

[14] L. Cao, W. Fei, H. Grosshans, and N. Cao, "Simulation of underwater explosions initiated by high-pressure gas bubbles of various initial shapes," Applied Sciences, vol. 7, no. 9, p. 880, 2017. 
[15] T. Li, S. Wang, S. Li, and A.-M. Zhang, "Numerical investigation of an underwater explosion bubble based on FVM and VOF," Applied Ocean Research, vol. 74, pp. 49-58, 2018.

[16] Y. M. Zhang, R. Tang, Q. Li et al., "The low-frequency sound power measuring technique for an underwater source in a non-anechoic tank," Measurement Science and Technology, vol. 29, no. 3, Article ID 035101, 2018.

[17] W. D. Song, M. H. Hong, B. Lukyanchuk, and T. C. Chong, "Laser-induced cavitation bubbles for cleaning of solid surfaces," Journal of Applied Physics, vol. 95, no. 6, pp. 2952-2956, 2004.

[18] G. L. Chahine, A. Kapahi, J.-K. Choi, and C.-T. Hsiao, "Modeling of surface cleaning by cavitation bubble dynamics and collapse," Ultrasonics Sonochemistry, vol. 29, pp. 528-549, 2016.

[19] Q. Li, J. Song, and D. Shang, "Experimental investigation of acoustic propagation characteristics in a fluid-filled polyethylene pipeline," Applied Sciences, vol. 9, no. 2, p. 213, 2019.

[20] E. Iran and N. Lima, "Modeling the liquid volume flux in bubbly jets using a simple integral approach," Journal of Hydraulic Engineering, vol. 138, no. 2, pp. 210-215, 2012.

[21] B. B. Wang and S. A. Socolofsky, "On the bubble rise velocity of a continually released bubble chain in still water and with crossflow," Physics of Fluids, vol. 27, no. 10, pp. 1-20, 2015.

[22] W. Bragg, The World of Sound, G. Bell \& Sons Ltd., London, UK, 1920 .

[23] M. Minnaert, "XVI.On musical air-bubbles and the sounds of running water," The London, Edinburgh, and Dublin Philosophical Magazine and Journal of Science, vol. 16, no. 104, pp. 235-248, 1933.

[24] C. Devin, "Survey of thermal, radiation, and viscous damping of pulsating air bubbles in water," The Journal of the Acoustical Society of America, vol. 31, no. 12, pp. 1654-1667, 1959.

[25] A. M. Worthington, A Study of Splashes, Longman's Green and Co., Inc., New York, NY, USA, 1908.

[26] G. J. Franz, "Splashes as sources of sound in liquids," The Journal of the Acoustical Society of America, vol. 31, no. 6, pp. 1080-1096, 1959.

[27] F. Laville, "Underwater sound generation by rainfall," The Journal of the Acoustical Society of America, vol. 82, no. 9, pp. 715-721, 1991.

[28] M. J. Buckingham, "On acoustic transmission in ocean-surface waveguides," Philosophical Transactions of the Royal Society of London. Series A: Physical and Engineering Sciences, vol. 335, no. 1639, pp. 513-555, 1991.

[29] H. C. Pumphrey, L. A. Crum, and L. Bjorno, "Underwater sound produced by individual drop impacts and rainfall," The Journal of the Acoustical Society of America, vol. 85, no. 4, pp. 1518-1526, 1989.

[30] H. Medwin, A. Kurgan, and J. A. Nystuen, "Impact and bubble sound from raindrops at normal and oblique incidence," The Journal of the Acoustical Society of America, vol. 88, no. 1, pp. 413-418, 1990.

[31] H. Medwin, J. A. Nystuen, P. W. Jacobus, L. H. Ostwald, and D. E. Snyder, "The anatomy of underwater rain noise," The Journal of the Acoustical Society of America, vol. 92, no. 3, pp. 1613-1623, 1992.

[32] J. A. Nystuen and H. Medwin, "Underwater sound produced by rainfall: secondary splashes of aerosols," The Journal of the Acoustical Society of America, vol. 97, no. 3, pp. 1606-1613, 1995.

[33] M. S. Longuet-Higgins, "An analytic model of sound production by raindrops," Journal of Fluid Mechanics, vol. 214, no. 1, pp. 395-410, 1990.
[34] H. N. Oguz and A. Prosperetti, "Bubble entrainment by the impact of drops on liquid surfaces," Journal of Fluid Mechanics, vol. 219, no. -1, pp. 143-163, 1990.

[35] H. Medwin and M. Beaky, "Bubble sources of the Knudsen sea noise spectra," Journal of the Acoustical Society of America, vol. 86, no. 6, pp. 1124-1130, 1989.

[36] C. S. Clay and H. Medwin, Acoustical Oceanography, John Wiley \& Sons, Inc., New York, NY, USA, 1977.

[37] S. Dajing, L. Qi, S. Dejiang et al., "Measurement of the radiated power of sound sources in a reverberation pool," Journal of Harbin Engineering University, vol. 31, no. 7, pp. 938-944, 2010, in Chinese.

[38] G. Gillot, C. Derec, J.-M. Génevaux, L. Simon, and L. Benyahia, "A new insight on a mechanism of airborne and underwater sound of a drop impacting a liquid surface," Physics of Fluids, vol. 32, no. 6, Article ID 062004, 2020.

[39] M. Strasberg, "The pulsation frequency of nonspherical gas bubbles in liquids," The Journal of the Acoustical Society of America, vol. 25, no. 3, pp. 536-537, 1953. 